\title{
A Review on Mode of Delivery during COVID-19 between December 2019 and April 2020
}

\author{
Margot L. Debrabandere, BA ${ }^{1}$ Dana C. Farabaugh, $\mathrm{MD}^{2} \quad$ Carolyn Giordano, $\mathrm{PhD}^{1}$ \\ ${ }^{1}$ Department of Medical Education, Drexel University College of \\ Medicine, Philadelphia, Pennsylvania \\ 2 Department of Obstetrics and Gynecology, Drexel University College \\ of Medicine, Philadelphia, Pennsylvania \\ Address for correspondence Margot Debrabandere, BA, C/O Dana \\ Farabaugh, Suite 114K, 2900 West Queen Lane, Philadelphia, \\ PA 19129 (e-mail: md3476@drexel.edu). \\ Am J Perinatol 2021;38:332-341.
}

\begin{abstract}
Keywords

- COVID-19

- pregnancy

- cesarean delivery

- vaginal delivery

- neonatal outcome

- vertical transmission

- maternal mental health

Objective This study aims to review the published literature to determine mode of delivery in pregnant women with coronavirus disease 2019 (COVID-19) and the indications reported for cesarean section early in the pandemic to add information to the current narrative and raise awareness of trends discovered.

Study Design A systematic review was conducted by searching PubMed, Scopus, and ScienceDirect databases for articles published between December 2019 and April 29, 2020 using a combination of the keywords such as COVID-19, coronavirus 2019, severe acute respiratory syndrome coronavirus 2 (SARS-CoV-2), pregnancy, vaginal delivery, cesarean section, vertical transmission, management, and guidelines. Peer-reviewed case studies with confirmed SARS-CoV-2 women who delivered were included to determine mode of delivery, indications for cesarean section, and maternal and neonatal characteristics.

Results A review of 36 total articles revealed deliveries in 203 SARS-CoV-2 positive pregnant women. A comparable severity of disease in pregnant versus nonpregnant women was noted, as previously determined. Overall, $68.9 \%$ of women delivered via cesarean section, with COVID-19 status alone being a common indication. Maternal COVID-19 may also be associated with increased risk of preterm labor, although neonatal outcomes were generally favorable. Despite eight of 206 newborns testing positive for SARS-CoV-2, there remains no definitive evidence of vertical transmission. Conclusion COVID-19 status alone became a common indication for cesarean delivery early in the pandemic, despite lack of evidence for vertical transmission. The increase in cesarean rate in this data may reflect obstetricians attempting to serve their patients in the best way possible given the current climate of constantly evolving guidelines on safest mode of delivery for the mother, infant, and provider. Upholding current recommendations from trusted organizations as new data are published, while also providing individualized support to expecting mothers on most appropriate mode of delivery, will reduce the amount of unnecessary, unplanned cesarean sections and could lessen the psychological impact of delivering during the COVID-19 pandemic.
\end{abstract}

received

May 15, 2020

accepted after revision

November 4, 2020

published online

December 7, 2020 (c) 2020. Thieme. All rights reserved.

Thieme Medical Publishers, Inc.,

333 Seventh Avenue, 18th Floor,

New York, NY 10001, USA
DOI https://doi.org/

10.1055/s-0040-1721658.

ISSN 0735-1631. 


\section{Key Points}

- COVID-19 may result in an increased rate of cesarean delivery for SARS-CoV-2 positive pregnant women.

- COVID-19 is a commonly reported indication for cesarean section, despite management guidelines urging against this.

- Although eight neonates tested positive for SARS-CoV-2, all additional fluid and tissue samples tested negative.

Coronavirus disease 2019 (COVID-19), caused by severe acute respiratory syndrome coronavirus 2 (SARS-CoV-2), emerged in late December 2019 when patients with a pneumonia of unknown etiology were admitted to hospitals in Wuhan, the capital of the Hubei Province in China. ${ }^{1}$ January 21, 2020 marked the first case in the United States, and by March 11, 2020, the World Health Organization (WHO) declared the virus a pandemic. ${ }^{1}$ The state of knowledge on coronavirus remains in flux and is continuously evolving.

Coronaviruses are a family of enveloped, positive-sense, single-stranded RNA viruses. ${ }^{1}$ Although four human coronaviruses are known to cause a mild and common cold, three human coronaviruses cause more severe disease, including SARS-CoV, Middle East respiratory syndrome coronavirus (MERS-CoV), and the novel SARS-CoV-2 that causes COVID-

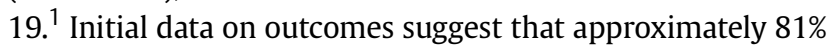
of COVID-19 cases are mild, $14 \%$ are severe, and $5 \%$ of patients become critically ill. ${ }^{2}$ The WHO estimates the global mortality rate of SARS-CoV-2 to be $3.4 \%$, although additional reports suggest that the mortality rate may be lower at $1.4 \%{ }^{3}$ In comparison, SARS-CoV and MERS-CoV have mortality rates of 10 and $37 \%$, respectively. ${ }^{3}$

Pregnant women may be particularly vulnerable to respiratory pathogens and severe pneumonia due to their modulated immune and cardiopulmonary systems. Of note, viral pneumonia is one of the leading causes of pregnancy deaths globally. ${ }^{1}$ Alterations in cell-mediated immunity play a central role in this susceptibility, as this enables the pregnant woman to remain tolerant to the allogeneic fetus, but decreases her ability to defend against intracellular pathogens such as viruses. ${ }^{1}$ In addition, the physiologic changes that occur in the cardiopulmonary systems during pregnancy, including increased oxygen consumption, decreased total lung volumes, diaphragm elevation due to the gravid uterus, and vasodilation leading to increased mucosal edema and secretions in the upper respiratory tract, cause the pregnant woman to be more intolerant to hypoxia. ${ }^{4}$ The impact of viral pneumonia during pregnancy was evident during the 1918 influenza pandemic, which caused a mortality rate of $37 \%$ among pregnant women, but only $2.6 \%$ in the general population. ${ }^{3}$ In addition, the H1N1 influenza pandemic in 2009 saw a higher rate of hospital admission for pregnant women than the general population. ${ }^{3}$ The 2002 SARS-CoV pandemic caused $50 \%$ of infected pregnant women to be admitted to an intensive care unit (ICU), with $33 \%$ requiring mechanical ventilation, and $25 \%$ dying due to SARS-CoV complications. ${ }^{3}$ Lastly, during the MERS-CoV outbreak of 2012, 63.6\% required ICU admission, with a case-fatality rate in pregnant women of $35 \%{ }^{5}$

The knowledge gained from these viral outbreaks has likely influenced the management of pregnant women dur- ing COVID-19. The purpose of this review is to present information with special focus on mode of delivery in SARS-CoV-2 positive pregnant women, indications reported for cesarean section, immediate neonatal outcomes, risk of vertical transmission, and the impact on maternal mental health. This examination will offer information that may help direct clinical practice within labor and delivery.

\section{Materials and Methods}

\section{Literature Search}

A systematic literature search was conducted by using PubMed, Scopus, and ScienceDirect databases for articles published between December 2019 and April 29, 2020. Combinations of the following search terms were used: COVID-19, coronavirus 2019, SARS-CoV-2, pregnancy, vaginal delivery, cesarean section, vertical transmission, management, and guidelines. Reference lists of large systematic reviews were also reviewed to ensure inclusion of other pertinent studies. Several case reports and case series were analyzed for inclusion. It is important to note that reporting bias may be present in the literature as adverse maternal and neonatal outcomes seen during the COVID-19 pandemic may have a greater tendency to be reported.

\section{Selection of Studies}

Inclusion criteria included studies reporting original data, laboratory-confirmed COVID-19 infection using quantitative real-time polymerase chain reaction (qRT-PCR), SARS-CoV-2 infected pregnant women who delivered, and availability of clinical characteristics, including maternal, pregnancy, and neonatal outcomes. Exclusion criteria included literature or systematic reviews, reports that were not peer-reviewed or in any language other than English, suspicion of duplicate reporting, suspected cases of COVID-19 that were not deemed confirmed, unreported maternal or neonatal outcomes, and pregnant women who did not deliver. Five studies included a population of pregnant women, including those who delivered and those who continued their pregnancy. If the outcomes of those who delivered were reported clearly, the study was included, but excluded those who did not deliver in the data collection.

\section{Data Extraction}

A patient, intervention, comparator, outcome, and study (PICOS) design structure was used to establish the study question, inclusion criteria, and data extraction points ( - Table 1 ). The main study question was: "What is the most common mode of delivery and indication for cesarean section reported, in addition to maternal and neonatal clinical outcomes, in SARS-CoV-2 positive pregnant women who delivered?" 


\begin{tabular}{l} 
Table 1 Patient, intervention, comparator, outcome, and study design structure for inclusion criteria and data extraction of \\
studies \\
$\begin{array}{lll}\text { Parameter } & \text { Inclusion criteria } & \text { Data extraction } \\
\text { Patient } & \text { SARS-CoV-2 infected } \\
\text { pregnant women who deliver } & \begin{array}{l}\text { Age, gestational age, severity of COVID-19, medical comorbidities, } \\
\text { pregnancy complications, ICU admission, and maternal mortality }\end{array} \\
\begin{array}{l}\text { Intervention } \\
\text { Comparator }\end{array} & \text { Delivery } & \text { Mode of delivery and indication for cesarean section } \\
\text { Outcome } & \text { None } & \begin{array}{l}\text { Preterm delivery, Apgar score, NICU transfer, } \\
\text { intrauterine or neonatal death, SARS-CoV-2 positivity }\end{array} \\
\text { Study } & \text { Case reports and case series } & \text { Type of study design }\end{array}$ \\
\hline
\end{tabular}

Abbreviations: NICU, neonatal intensive care unit; SARS-CoV-2, severe acute respiratory syndrome coronavirus 2.

\section{Data Synthesis}

The main outcomes assessed were frequency of cesarean section for COVID-19 status alone, vaginal delivery, Apgar score $<7$, preterm birth ( $<37$ weeks), neonatal intensive care unit (NICU) transfer, fetal and neonatal death, and neonatal SARS-CoV-2 infection. A descriptive summary was used to present these results, organized by maternal clinical characteristics, mode of delivery, and neonatal clinical characteristics. A limitation of this review is its sample size and the lack of statistical analysis. A large, prospective, and randomized-controlled study would need to be conducted to allow for robust statistical significance of results. All study investigators (M.L.D., D.C.F., and C.G.) independently reviewed the data collection forms to verify data accuracy.

\section{Quality Assessment}

Two independent examiners (M.L.D. and D.C.F.) applied the guidelines of the Preferred Reporting Items for Systematic Reviews and Meta-Analyses (PRISMA) for the data extraction and quality assessment. The examiners (M.L.D. and D.C.F.) independently assessed the methodologies of the studies according to the tool for evaluating the methodological quality of case reports and case series described by Murad et al. ${ }^{6}$ The tool considers four domains (selection, ascertainment, causality, and reporting) and provides eight questions to aid quality score. If all domains were satisfied, the study would be classified as "good quality"; if three of the domains were satisfied, the study would be classified as "fair quality"; and if only two or one of the domains were satisfied, the study would be classified as "poor quality." Precisely, 18 studies fulfilled all of the domains and were judged to be of good quality, 13 studies were classified as fair quality, and 5 studies were judged to be of poor quality.

\section{Results}

After exclusion of contents not related to the topic of our review, duplicates, review papers or guidelines, studies published without peer review, and studies published in alternate languages, including Chinese, German, and French, a total of 36 original studies were reviewed and included ( - Table 2). - Fig. 1 shows the process of study inclusion for the systematic review. These studies include one case-control study from China, ${ }^{7} 18$ case reports (from China, ${ }^{8-14}$

\begin{tabular}{|c|c|c|c|}
\hline Study design & Country & $\begin{array}{l}\text { Count of } \\
\text { reports }\end{array}$ & $\begin{array}{l}\text { Sum of pregnant } \\
\text { women with } \\
\text { COVID- } 19 \text { who } \\
\text { deliver }\end{array}$ \\
\hline Case-control & China & 1 & 16 \\
\hline \multirow{10}{*}{ Case report } & China & 7 & 7 \\
\hline & USA & 3 & 3 \\
\hline & Australia & 1 & 1 \\
\hline & Korea & 1 & 1 \\
\hline & Iran & 1 & 1 \\
\hline & Honduras & 1 & 1 \\
\hline & Sweden & 1 & 1 \\
\hline & Turkey & 1 & 1 \\
\hline & Spain & 1 & 1 \\
\hline & Peru & 1 & 1 \\
\hline \multirow[t]{5}{*}{ Case series } & China & 11 & 93 \\
\hline & USA & 3 & 25 \\
\hline & Italy & 1 & 42 \\
\hline & Iran & 1 & 7 \\
\hline & $\begin{array}{l}\text { Canada } \\
\text { and } \\
\text { France }\end{array}$ & 1 & 2 \\
\hline Total & & 36 & 203 \\
\hline
\end{tabular}

${ }^{\mathrm{a}}$ Two cases reported in same series due to similar complication; one patient from each country.

United States, ${ }^{15-17}$ Australia, ${ }^{18}$ Korea, ${ }^{19}$ Iran, ${ }^{20}$ Honduras, ${ }^{21}$ Sweden, ${ }^{22}$ Turkey, ${ }^{23}$ Spain, ${ }^{24}$ and Peru, ${ }^{25}$ ), and 18 retrospective case series (from China, ${ }^{4,26-35}$ United States, ${ }^{36-38}$ Italy, ${ }^{39}$ Iran, ${ }^{40}$ Canada, and France ${ }^{41}$ ).

A total of 203 SARS-CoV-2 positive pregnant women who delivered were reported in the studies. One patient was confirmed by the local Centers for Disease Control and Prevention (CDC) once additional illnesses were excluded, as the patient presented with typical symptoms and evidence of a viral interstitial pneumonia on computerized tomography (CT) scan, despite lack of a positive SARS-CoV-2 RT-PCR test. ${ }^{35}$ This patient was included in this review, as she was deemed confirmed. All additional suspected COVID-19 cases that 


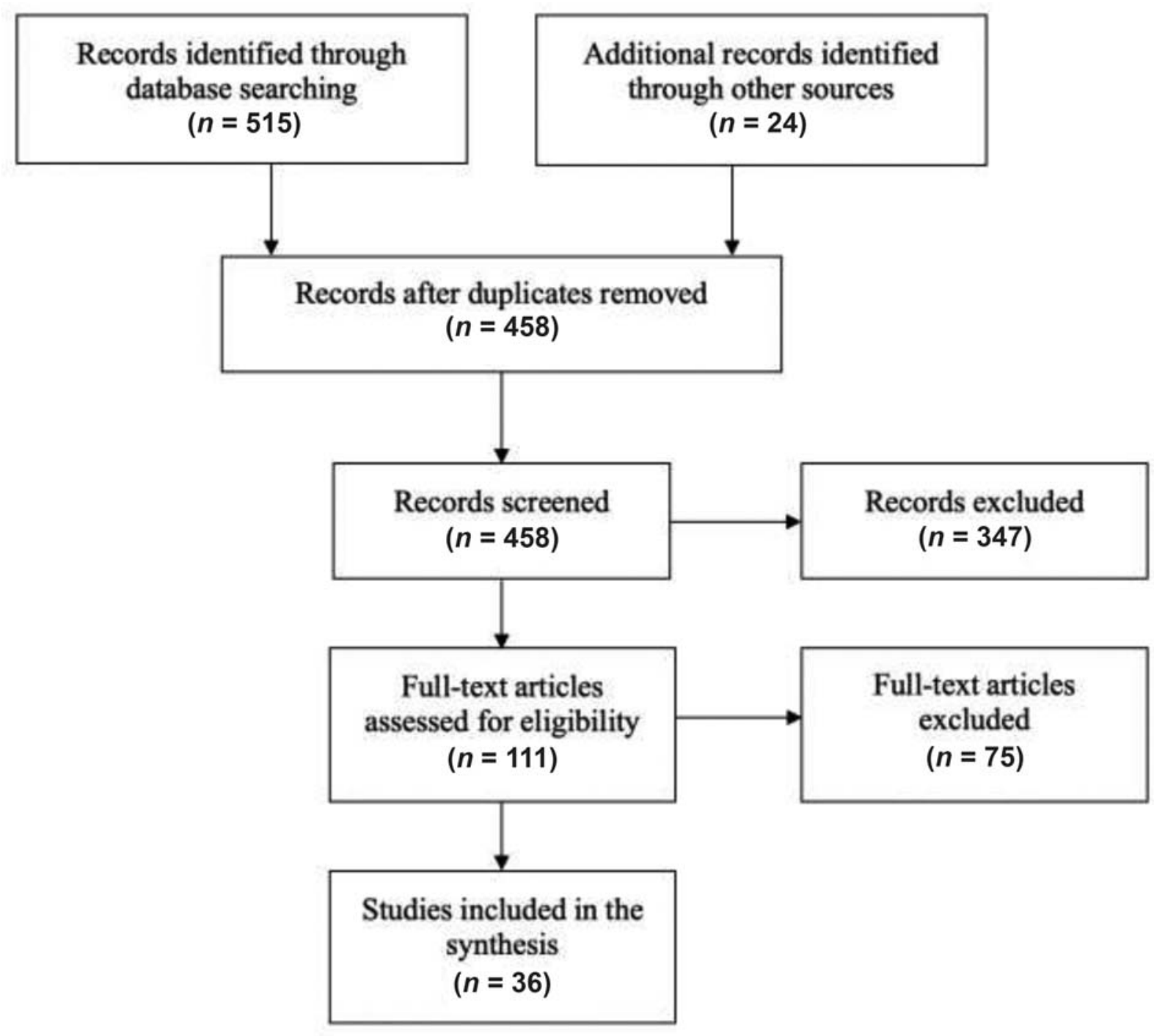

Fig. 1 Study flow chart.

were not deemed confirmed were excluded. Because six twin deliveries occurred, a total of 206 neonates were reported.

\section{Maternal Clinical Characteristics}

The maternal age ranged from 20 to 49 years old. The gestational age of the women who delivered ranged from $28^{0 / 7}$ to $41^{2 / 7}$ weeks ( - Table 3 ).

Of the 203 pregnant women who delivered, 30 (14.8\%) suffered severe SARS-CoV-2 disease, while the remaining 173 (85.2\%) women had nonsevere disease, including an asymptomatic, mild, or moderate COVID-19 course. Twenty-one (10.3\%) women with severe disease were admitted to an intensive care unit (ICU), ten (4.93\%) of whom remained in the hospital at the time of the case report or series being published. Six (2.96\%) maternal mortalities in women who delivered were reported. Of those, Karami et $\mathrm{al}^{20}$ reported one maternal mortality, although the cause of death was deemed uncertain, with acute respiratory distress syndrome (ARDS), alveolar hemorrhage, and acute collagen vascular autoimmune disease on the differential diagnosis, and Hantoushzadeh et $\mathrm{al}^{40}$ reported five maternal mortalities, all of whom suffered a combination of ARDS, cardiopulmonary collapse, disseminated intravascular coagulation, septic shock, and end-organ failure. Of note, Hantoushzadeh et $\mathrm{al}^{40}$ study reported only adverse maternal outcomes, which may have added reporting bias to this literature review.

Maternal comorbidities were diverse, with obesity, hypothyroidism, mild intermittent asthma, polycystic ovary syndrome, pregestational diabetes mellitus, chronic hypertension, and uterine scarring being most common, as seen in -Table 3 . Several pregnancy complications were also reported, with gestational diabetes mellitus, premature rupture of membranes, preeclampsia, gestational hypertension, episodes of vaginal bleeding, including placental abruption, and twin pregnancy being most common.

Mode of Delivery and Indication for Cesarean Section In total, 140 of the 203 (68.9\%) women were delivered by cesarean section, while the remaining 63 (31\%) women were reported to have a successful vaginal delivery. Because 
Table 3 Maternal characteristics and clinical outcomes of COVID-19 infection in pregnant women

\begin{tabular}{|c|c|}
\hline & $\begin{array}{l}\text { All mothers } \\
(n=203)\end{array}$ \\
\hline \multicolumn{2}{|l|}{ Maternal characteristics } \\
\hline Age range $(y)$ & $20-49$ \\
\hline Gestational age (wk) & $28^{0 / 7}-41^{2 / 7}$ \\
\hline Positive RT-PCR for SARS-CoV-2 & $202^{\mathrm{a}}$ \\
\hline \multicolumn{2}{|l|}{ Maternal disease severity } \\
\hline $\begin{array}{l}\text { Nonsevere (asymptomatic, } \\
\text { mild, moderate) }\end{array}$ & 173 \\
\hline Severe & 30 \\
\hline \multicolumn{2}{|l|}{ Maternal comorbidities } \\
\hline Obesity & 33 \\
\hline Hypothyroidism & 9 \\
\hline Mild intermittent asthma & 8 \\
\hline Polycystic ovarian syndrome & 4 \\
\hline Type 2 diabetes mellitus & 4 \\
\hline Chronic hypertension & 4 \\
\hline Uterine scarring & 4 \\
\hline Anemia & 4 \\
\hline Chronic hepatitis B & 2 \\
\hline Valvular replacement surgery & 1 \\
\hline $\begin{array}{l}\text { Myotonic dystrophy, bicuspid } \\
\text { aortic valve, mild CVA on OCP }\end{array}$ & 1 \\
\hline Familial neutropenia & 1 \\
\hline Sinus tachycardia & 1 \\
\hline \multicolumn{2}{|l|}{ Pregnancy complications } \\
\hline Gestational diabetes mellitus & 19 \\
\hline PPROM & 8 \\
\hline Preeclampsia & 8 \\
\hline PROM & 6 \\
\hline Gestational hypertension & 7 \\
\hline $\begin{array}{l}\text { Episode of vaginal bleeding } \\
\text { (including placental abruption) }\end{array}$ & 4 \\
\hline Twin pregnancy & 3 \\
\hline Placenta previa & 3 \\
\hline History of stillbirth & 2 \\
\hline Acute coagulopathy and transaminitis & 2 \\
\hline Amniotic fluid abnormality & 2 \\
\hline Umbilical cord abnormality & 2 \\
\hline Cholestasis of pregnancy & 1 \\
\hline Focal accreta & 1 \\
\hline Influenza & 1 \\
\hline \multicolumn{2}{|l|}{ Maternal clinical outcomes } \\
\hline Intensive care unit & 21 \\
\hline Remaining in hospital & 10 \\
\hline Maternal mortality & 6 \\
\hline
\end{tabular}

Abbreviations: CVA, cerebrovascular accident; OCP, oral contraceptives; PPROM, preterm premature rupture of membranes; PROM, premature rupture of membranes; RT-PCR, real-time transcriptase polymerase chain reaction; SARS-CoV-2, severe acute respiratory syndrome coronavirus 2 . ${ }^{\mathrm{a}}$ One patient deemed confirmed by local CDC without a positive SARSCoV-2 result.
Table 4 Mode of delivery and indication for cesarean section

All mothers $(n=203)$

Delivery characteristics

Cesarean section $\quad 140$

Vaginal delivery 63

Indication for cesarean delivery

Obstetric indication 65

Fetal distress 16

Nonreassuring fetal heart rate tracing 11

PPROM 8

Repeat cesarean delivery 7

Severe preeclampsia 5

Decreased fetal movement 5

Placenta previa 3

Scheduled cesarean delivery ${ }^{\mathrm{a}} \quad 2$

Placental abruption 2

Umbilical cord abnormality 2

Arrest of descent 1

Arrest of dilation 1

Failed labor induction $\quad 1$

Obstructed labor with incomplete $\quad 1$ rotation of fetal head

No indication provided 39

COVID-19 status alone $\quad 38$

Worsening maternal status $\quad 22$

due to COVID-19

History of stillbirth or 2

stillbirth of current fetus

Abdominal pain

1

Severely elevated AST/ALT

1

Abbreviations: ALT, alanine aminotransferase; AST, aspartate aminotransferase; PPROM, preterm premature rupture of membranes.

${ }^{\mathrm{a}}$ For large for gestational age and twin pregnancy.

several women had multiple indications reported for cesarean section, each indication was added as a separate tally to provide a thorough depiction of the indications used to justify cesarean section. A total of 168 indications for the 140 women who delivered via cesarean section were reported. There were 65 women $(65 / 168,38.7 \%)$ with an obstetric indication for cesarean section ( - Table 4$)$. These obstetric indications included fetal distress or decreased fetal movement, nonreassuring fetal heart rate tracing, preterm premature rupture of membranes (PPROM), repeat or scheduled cesarean section, severe preeclampsia, placental or umbilical cord abnormalities, and failed labor induction. No indication for cesarean section was reported in 39 cases (39/168, 23.2\%), and the true indication for their cesarean section remains unknown. Maternal COVID-19 status was reported as an indication in 38 cases (22.6\%). Many studies note that COVID-19 status alone was an indication for 
cesarean section within their hospital due to concerns of vertical transmission or desire to treat the mother with antiviral therapy promptly without exposing the fetus. $^{4,7,10,27,33}$ If a worsening maternal status due to COVID-19 was reported as an indication for cesarean section, this was listed separately from the indication of COVID-19 as a diagnosis. Worsening maternal status as an indication was reported in 22 women $(22 / 168,13.1 \%)$. Although a compromised or decompensating cardiopulmonary status was most common, Juusela et $\mathrm{al}^{37}$ and Vlachodimitropoulou et $\mathrm{al}^{41}$ reported unique cases of acute coagulopathy with transaminitis and development of cardiomyopathy, respectively, as causes of maternal clinical decline and reason for cesarean intervention. Two patients had a history of stillbirth or stillbirth of the current fetus (2/168, 1.2\%). Abdominal pain was cited as an indication for one patient, without further elaboration (1/168, 0.6\%). Lastly, one patient had severely elevated transaminases, although it is unclear whether this was related to COVID-19 (1/168, 0.6\%).

\section{Neonatal Clinical Characteristics}

Preterm birth before 34 weeks of gestation was observed in 19 of the 206 (9.2\%) neonates, with the earliest delivery being at $28^{0 / 7}$ weeks of gestational age. This value includes three sets of twins. Preterm birth between 34 and $36^{6 / 7}$ weeks of gestational age occurred in 43 (20.9\%) neonates, with two sets of twins included in this value. In total, 62 (30.1\%) infants were preterm, and of those that were clearly indicated, spontaneous preterm birth occurred in 19 (9.2\%).

Of the 199 neonates with a reported Apgar score, the large majority were greater than or equal to seven. The lower range of Apgar scores reported in -Table 5 can be attributed to four infants. Ferrazzi et al $^{39}$ reported two preterm infants born before 34 weeks of gestational age with a 5-minute Apgar score $<7$, but do not indicate the severity of maternal disease. Kelly et al ${ }^{16}$ reported an infant born at 33 weeks of gestational age with Apgar scores of 1, 6, and 7 at 5, 10, and 15 minutes, respectively. The mother suffered critical COVID-19 that required ICU admission with intubation for 11 days. Lastly, Vlachodimitropoulou et $\mathrm{al}^{41}$ reported one infant born at $35^{5 / 7}$ weeks of gestational age with Apgar scores of 4, 2, and 7 at 1, 5, and 10 minutes, respectively, and this mother suffered severe COVID-19 complicated by progressive coagulopathy and transaminitis.

Total 26 of the 206 (12.6\%) neonates were transferred to the NICU. This value includes infants with complications and excludes those infants transferred to the NICU merely for isolation. These complications involved prematurity, a congenital multicystic dysplastic kidney, low-grade fever, respiratory distress, mild pneumonia, lymphopenia, and precautionary intubation due to high level of maternal sedation from severe maternal COVID-19 pneumonia. Zhu et al ${ }^{35}$ report a series of 10 neonates, each of which was admitted to the NICU for symptomatic supportive treatments. Notably, two of these infants developed severe disease requiring transfusion of platelets, plasma, red blood cells, and gamma globulin. One infant developed refractory shock with gastric bleeding, multiple organ failure, DIC, and death, while the second infant developed gastrointestinal hemorrhage and DIC, but recovered. All
Table 5 Preterm delivery and neonatal outcomes of COVID-19

All neonates $(n=206)$

Preterm delivery

Before 34 weeks

19 (three set of twins)

$34-36^{6 / 7}$ weeks 43 (two set of twins)

Neonatal outcomes

Apgar score range

Positive RT-PCR

for SARS-CoV-2

NICU transfer

(for issue, not isolation)

Intrauterine fetal death

Neonatal death $1-10$

8

26

Abbreviations: NICU, neonatal intensive care unit; RT-PCR, real-time transcriptase polymerase chain reaction; SARS-CoV-2, severe acute respiratory syndrome coronavirus 2 .

infants who tested positive for SARS-CoV-2 were sent to the NICU, whether the infant was asymptomatic and required isolation or had symptoms of respiratory distress.

There were four cases of intrauterine fetal death and three cases of neonatal death, including one set of twins. Intrauterine fetal death was reported only with critical maternal COVID-19, including a patient with ARDS and multiple organ dysfunction syndrome requiring extracorporeal membrane oxygenation support. ${ }^{31}$ Hantoushzadeh et $\mathrm{al}^{40}$ reported neonatal death on day of life three in a set of twins due to complications relating to preterm delivery at $28^{0 / 7}$ weeks of gestational age. These two infants were negative for SARS$\mathrm{CoV}-2$. Zhu et $\mathrm{al}^{35}$ report a neonate born at $34^{5 / 7}$ weeks of gestational age with an Apgar score of eight at 5 minutes that developed shortness of breath and moaning, thrombocytopenia, and abnormal liver function, which progressed to refractory shock, multiple organ failure, DIC, and death on day 9 of life. This newborn was also SARS-CoV-2 negative.

Lastly, the available literature has found no clear evidence for vertical transmission of COVID-19 from mother to fetus despite eight of the 206 (3.9\%) neonates in this review testing positive for SARS-CoV-2. Yu et $\mathrm{al}^{34}$ reported one infant with a positive SARS-CoV-2 nasopharyngeal swab at 36 hours of life, but with negative viral nucleic acid tests of the placenta and cord blood. This neonate was delivered via cesarean section and developed mild shortness of breath symptoms with chest X-ray revealing mild pulmonary infection. The symptoms resolved quickly with neonatal care. Ferrazzi et $\mathrm{al}^{39}$ reported three neonates with a positive SARS-CoV-2 test. Two of these neonates were found to be positive at days 1 and 3 after their mothers were diagnosed with SARSCoV-2 postpartum and breastfed without wearing a surgical mask. The third positive neonate was delivered vaginally at term in good condition to a mother wearing a surgical mask and was immediately separated due to maternal postpartum hemorrhage. Within several hours of delivery, gastrointestinal symptoms developed, but SARS-CoV-2 testing returned equivocal. Three days later, respiratory symptoms began, and 
a SARS-CoV-2 test returned positive. This neonate recovered after 1 day of mechanical ventilation and remains in good condition. Dong et $\mathrm{al}^{8}$ reported an infant girl delivered via cesarean section without skin-to-skin contact and to a mother wearing a N-95 mask, who tested negative for SARS-CoV-2 at 2 hours to 16 days of life, but had elevated levels of both SARS-CoV-2 IgG and IgM levels at 2 hours of life. These remained elevated for 14 days, in addition to an elevated IL-6, IL-10, and white blood cell count. This neonate remained asymptomatic and with a negative chest CT. Hantoushzadeh et $\mathrm{al}^{40}$ reported one neonate delivered via cesarean section at $30^{5 / 7}$ weeks of gestational age testing negative for SARS-CoV-2 at birth, but positive at day 7 while intubated in the NICU for prematurity and pneumonia. This patient has recovered and is stable. Díaz et $\mathrm{al}^{24}$ reported one neonate delivered via cesarean section to a mother who developed symptoms 3 days postpartum and was diagnosed with COVID-19 5 days postpartum. This neonate stayed with her mother in the maternity ward, and despite a negative SARS-CoV-2 result at 6 days of life, the infant was positive on day 8 . This infant became mildly symptomatic with resolution 24 hours later. Lastly, Alzamora et $\mathrm{al}^{25}$ reported a neonate delivered via cesarean section positive for SARS-CoV-2 at 16 and 48 hours of life, although with negative IgG and IgM levels. Delayed cord clamping and skin-to-skin were not performed, and the infant was immediately separated from mother after birth. This neonate became mildly symptomatic on day 6 of life and was adequately supported with supplemental oxygen via nasal cannula.

\section{Discussion}

In summary, the clinical characteristics of SARS-CoV-2 positive pregnant women who delivered in this review were similar to those of nonpregnant SARS-CoV-2 positive patients, as reported by Wu et al. ${ }^{2}$ These results also seem to suggest that COVID-19 is less severe than SARS and MERS during pregnancy. ${ }^{1}$ Immediate neonatal outcomes were largely favorable, with the majority having an Apgar score of $\geq 7$, and generally 9 or 10 . Four infants (2\%) had a lower Apgar score due to being very preterm or having a mother in critical status. Precisely, $12.6 \%$ of neonates required transfer to the NICU, mainly for prematurity or respiratory distress, with the large majority having negative SARS-CoV-2 test results. Lastly, four intrauterine fetal deaths occurred in mothers with severe COVID-19 and three neonatal deaths occurred in SARS-CoV-2 negative neonates.

The rate of preterm birth (30.1\%) found in this study is high, with several of these preterm deliveries being the result of a cesarean intervention for a positive COVID-19 status. This demonstrates the negative impact that cesarean section may have on newborns during COVID-19. However, because spontaneous preterm labor occurred with a rate of $9.2 \%$, there may be an increased risk of spontaneous preterm birth in mothers with COVID-19 independent of decision for cesarean section. These rates of preterm birth found are roughly similar to what was observed during SARS-CoV and MERS-CoV, where two of 16 (12.5\%) and three of 11 (27.3\%) neonates were premature, respectively. ${ }^{42}$ Although sample size and lack of statistical analysis is a limitation in this comparison, it would be plausible to assume that severe coronaviruses may cause an increased rate of preterm delivery. ${ }^{42}$ Importantly, these rates for SARS-CoV, MERS-CoV, and SARS-CoV-2 are higher than the average rate of preterm delivery during nonpandemic times, which was approximately $10.6 \%$ worldwide in 2014 , with a range of $8.7 \%$ in European countries to $13.4 \%$ in Northern Africa. ${ }^{43}$

The uncertainty of vertical transmission is of particular interest and may be a contributing factor to the increased rate of cesarean section. Eight (3.9\%) neonates tested positive in this review, six of whom were born via cesarean section and two via vaginal delivery. Although the majority of cases contained a possible contact with an infected individual, cases with an uncertain origin for each mode of delivery remain. Within the cesarean section group, one infant tested SARS-CoV-2 positive at 36 hours, another at 16 and 48 hours despite negative $\operatorname{IgG}$ and IgM levels, and a third infant who was SARS-CoV-2 negative at 2 hours of age to 16 days, but positive for IgG and IgM beginning at 2 hours of life and continuing for 14 days. ${ }^{8,25,34}$ This last infant has a similar presentation to that seen for five of the six infants within Zeng et $\mathrm{al}^{44}$ study. These five newborns born via cesarean section tested negative for SARS-CoV-2, but presented with either elevated $\operatorname{IgG}$ and $\operatorname{IgM}$, or only $\operatorname{IgG}$, as well as elevated IL-6. Despite this, none of the infants became symptomatic. While passive transfer of mother's IgG across the placenta begins at the end of the second trimester, IgM is not typically transferred from mother to fetus due to its large molecular structure. Therefore, one rationale for this finding is that IgM may have been produced by the infant if the virus had crossed the placenta. Kimberlin et $\mathrm{al}^{45}$ discuss these findings of elevated IgM in neonates, and argue that IgM assays are not commonly used procedures for detecting congenital infections due to their susceptibility to false negatives and false positives, crossreactivity, and additional testing challenges. Consequently, while detection of IgM in the newborn is significant, caution must be exercised as these assays are often less reliable than nucleic acid amplification diagnostic tests. ${ }^{45}$ The remaining two SARS-CoV-2 positive infants were delivered vaginally, one of which had contact with their SARS-CoV-2 positive mother while breastfeeding without droplet precautions. The second neonate's clinical course is significant, as an equivocal test result was found a few hours after birth and a confirmed positive result on day $3 .^{39}$ The mother wore a surgical mask during labor and immediate separation of mother and newborn was enforced. Unfortunately, no samples of amniotic or vaginal fluid, placental tissue, or umbilical cord blood were tested for SARS-CoV-2 in this patient. These findings suggest that the possibility of in utero transmission is still present; however, not one case has proved the phenomenon.

Additional evidence against in utero transmission is the lack of positive SARS-CoV-2 in amniotic fluid, vaginal fluid, placental tissue, umbilical cord blood, and breast milk. In this literature review, only two of the eight SARS-CoV-2 positive neonates had additional samples taken from these sites. 
However, when performed, all tests returned negative. Qiu et $\mathrm{al}^{46}$ were unable to detect SARS-CoV-2 in the vaginal fluid of postmenopausal women with severe COVID-19, suggesting that vertical transmission through both cesarean section and vaginal delivery is low. This study was limited by the inability to use premenopausal women's vaginal swabs; however, it does provide useful information as both Zika and Ebola viruses were detected in the female reproductive tracts. Yu et al ${ }^{47}$ evaluated the amniotic fluid of two women infected with SARS-CoV-2 during the first trimester. These women underwent percutaneous ultrasound-guided amniocentesis once recovered from COVID-19 in their second trimesters. Neither SARS-CoV-2 nor IgG and IgM were detected, although the possibility of a transient elevation during infection-as seen with Zika virus-remains.

Despite the lack of evidence that SARS-CoV-2 can be transmitted from mother to fetus in utero, this review has determined that the rate of cesarean section is increased when compared with nonpandemic times. Overall, $68.9 \%$ of women were found to deliver via cesarean section, which aligns with results of the largest systematic review to date by Elshafeey et al. ${ }^{48}$ It is important to note that reporting bias may be influencing this percentage, as many pregnant women with COVID-19 have likely delivered their infant vaginally during this pandemic but without publication of the information. The results of a study examining mode of delivery in COVID-19 positive pregnant women in Spain conducted by Martínez-Perez et $\mathrm{al}^{49}$ also align with this overarching theme of an increased cesarean rate. Their results showed 41 (53\%) women delivering vaginally and $37(47 \%)$ women delivering by cesarean section. Those delivering via cesarean were more likely to be multiparous, obese, require oxygen at admission, and have abnormal chest X-ray findings than those delivering vaginally. Most notably, cesarean birth was significantly associated with maternal clinical deterioration, whereas no women with vaginal deliveries developed severe adverse outcomes. Martínez-Perez et $\mathrm{al}^{49}$ note that the physiological stress induced by surgery has been known to increase postpartum maternal complications.

Al-Tawfiq et $\mathrm{al}^{5}$ also determined that the rate of cesarean delivery during COVID-19 is higher than with MERS-CoV, where $40 \%$ delivered via cesarean section. Although the data for both of these coronaviruses are limited, the increased cesarean section rate with COVID-19 compared with MERS$\mathrm{CoV}$ is striking as outcomes in pregnant women with MERS$\mathrm{CoV}$ were much less favorable. This cesarean section rate is also higher than the global average rate, determined to be approximately $18.6 \%{ }^{50}$ This data included 150 countries from 1990 to 2014, and showed a range of cesarean section of 6 to $27.2 \%$ in the least and most developed countries, respectively. ${ }^{50}$ Ultimately, it is likely that during a pandemic with a novel virus and an uncertain risk of vertical transmission that the rate of cesarean section will be higher than during nonpandemic times. However, it is important to compare the various indications reported for this procedure to the suggested management guidelines to determine if they are acceptable.
The American College of Obstetricians and Gynecologists, the Royal College of Obstetricians and Gynecologists, and the International Society of Ultrasound in Obstetrics and Gynecology all advise that decision for cesarean delivery be individualized, dictated by obstetric indications, and not be influenced by COVID-19 status alone, as the likelihood of vertical transmission in utero is low. ${ }^{51-53}$ Based on this review, this recommendation was not upheld in $22.6 \%$ of cases. It is important to note that the majority of women with COVID-19 status alone as the indication for cesarean section were delivered in hospitals in China, where this was an initial guideline in the early stages of the pandemic. Severe COVID-19, with rapid maternal decompensation, including development of ARDS, septic shock, or acute organ failure, as well as fetal distress, as an indication for cesarean section occurred in $13.1 \%$ of women in this review. In these instances, guidelines recommend that a lower threshold for cesarean section be present, as delivery of the fetus may improve the mother's ventilation quickly, especially when $>34$ weeks of gestational age. ${ }^{52,53}$ Unfortunately, because $23.2 \%$ of women did not have an indication provided, the review's ability to offer a comprehensive understanding of the thought process of the practicing obstetricians and midwives is limited. Nonetheless, it is not only reassuring that the most commonly reported motive for cesarean section was an obstetrical issue or concern, but also that several case studies within this review described successful vaginal deliveries with adequate protection of staff, maternal, and neonatal well-being for asymptomatic, mild, and moderate COVID-19 cases.

In addition to following management guidelines on mode of delivery, incorporating the mother's preference for her delivery and minimizing the psychological impact of delivering during COVID-19 is crucial, as pregnancy is already known to be a time of increased vulnerability to changes in mental health. ${ }^{53}$ Saccone et $\mathrm{a}^{54}$ demonstrated that COVID19 has a moderate-to-severe psychological impact on pregnant women, with high levels of anxiety regarding possible vertical transmission. Wu et al ${ }^{55}$ also showed that after declaring COVID-19 an epidemic, significantly higher rates of depressive and anxiety symptoms, as well as increased thoughts of self-harm, were present in pregnant women when compared with pre-epidemic times. The extreme protective measures, such as home isolation and decreased in-person prenatal, intrapartum, and postnatal care and involvement from physicians and families, also likely contribute to this anxiety. ${ }^{56}$ Inclusion of the woman's preferences for mode of delivery while upholding accepted obstetric guidelines will help to support maternal mental health and minimize the psychological impact of needing to deliver during the COVID-19 pandemic.

\section{Conclusion}

This review aimed to provide information regarding mode of delivery and indications for cesarean section early in the COVID-19 pandemic. Maintaining an evidence-based approach during this time is critical when making clinical 
decisions on timing and mode of birth in SARS-CoV-2 positive pregnant women who become infected in the third trimester to minimize adverse outcomes. Given the lack of definitive evidence for increased risk of vertical transmission with a vaginal delivery in a SARS-CoV-2 positive mother with asymptomatic, mild, or moderate disease, it must be reiterated that COVID-19 status alone is not a contraindication to vaginal delivery. However, guidelines suggest that it is reasonable to lower the threshold for cesarean section with severe disease. If maternal or neonatal outcomes, including vertical transmission, or health care worker safety were to be compromised by vaginal delivery due to COVID-19, guidelines would need to adapt accordingly. This will require continued follow-up and careful reporting of maternal and neonatal outcomes. Continued research on pregnant women who suffer from COVID-19 during their first and second trimesters is also important to develop specific guidelines for this population, as they may differ from women infected in their third trimester. Lastly, adherence to guidelines from trusted organizations must be stressed and adequate, individualized care that emphasizes a mother's mental wellbeing and expectations for her pregnancy and childbirth experience must be guaranteed.

Conflict of Interest

None declared.

\section{References}

1 Schwartz DA, Graham AL. Potential maternal and infant outcomes from (Wuhan) coronavirus 2019-nCoV infecting pregnant women: lessons from SARS, MERS, and other human coronavirus infections. Viruses 2020;12(02):194

$2 \mathrm{Wu} \mathrm{Z}$, McGoogan JM. Characteristics of and important lessons from the coronavirus disease 2019 (COVID-19) outbreak in China: summary of a report of 72314 cases from the chinese center for disease control and prevention. JAMA 2020;323(13):1239-1242

3 Yan J, Guo J, Fan C, et al. Coronavirus disease 2019 in pregnant women: a report based on 116 cases. Am J Obstet Gynecol 2020; 223(01):111.e1-111.e14

4 Liu D, Li L, Wu X, et al. Pregnancy and perinatal outcomes of women with coronavirus disease (COVID-19) pneumonia: a preliminary analysis. AJR Am J Roentgenol 2020;215(01):127-132

5 Al-Tawfiq JA. Middle east respiratory syndrome coronavirus (MERS-CoV) and COVID-19 infection during pregnancy. Travel Med Infect Dis 2020;36:101641

6 Murad MH, Sultan S, Haffar S, Bazerbachi F. Methodological quality and synthesis of case series and case reports. BMJ Evid Based Med 2018;23(02):60-63

7 Li N, Han L, Peng M, et al. Maternal and neonatal outcomes of pregnant women with COVID-19 pneumonia: a case-control study. Clin Infect Dis 2020:ciaa352

8 Dong L, Tian J, He S, et al. Possible vertical transmission of SARSCoV-2 from an infected mother to her newborn. JAMA 2020;323 (18):1846-1848

9 Li Y, Zhao R, Zheng S, et al. Lack of vertical transmission of severe acute respiratory syndrome coronavirus 2, China. Emerg Infect Dis 2020;26(06):1335-1336

10 Lu D, Sang L, Du S, Li T, Chang Y, Yang XA. Asymptomatic COVID-19 infection in late pregnancy indicated no vertical transmission. J Med Virol 2020;1-5;

11 Peng Z, Wang J, Mo Y, et al. Unlikely SARS-CoV-2 vertical transmission from mother to child: a case report. J Infect Public Health 2020;13(05):818-820
12 Wang X, Zhou Z, Zhang J, Zhu F, Tang Y, Shen X. A case of 2019 novel coronavirus in a pregnant woman with preterm delivery. Clin Infect Dis 2020;71(15):844-846

13 Xia H, Zhao S, Wu Z, Luo H, Zhou C, Chen X. Emergency caesarean delivery in a patient with confirmed COVID-19 under spinal anaesthesia. Br J Anaesth 2020;124(05):e216-e218

14 Xiong $\mathrm{X}$, Wei $\mathrm{H}$, Zhang Z, et al. Vaginal delivery report of a healthy neonate born to a convalescent mother with COVID-19. J Med Virol 2020

15 Iqbal SN, Overcash R, Mokhtari N, et al. An uncomplicated delivery in a patient with COVID-19 in the United States. N Engl J Med 2020;382(16):e34

16 Kelly JC, Dombrowksi M, O'neil-Callahan M, Kernberg AS, Frolova AI, Stout MJ. False-negative COVID-19 testing: considerations in obstetrical care. Am J Obstet Gynecol MFM. 2020;100130;

17 Schnettler WT, Al Ahwel Y, Suhag A. Severe ARDS in COVID-19infected pregnancy: obstetric and intensive care considerations. Am J Obstet Gynecol MFM. 2020;100120;

18 Lowe B, Bopp B. COVID-19 vaginal delivery: a case report. Aust N Z J Obstet Gynaecol 2020;60(03):465-466

19 Lee DH, Lee J, Kim E, Woo K, Park HY, An J. Emergency cesarean section on severe acute respiratory syndrome coronavirus 2 (SARS-CoV-2) confirmed patient. Korean J Anesthesiol 2020. Doi: $10.4097 /$ kja.20116

20 Karami P, Naghavi M, Feyzi A, et al. WITHDRAWN: mortality of a pregnant patient diagnosed with COVID-19: a case report with clinical, radiological, and histopathological findings. Travel Med Infect Dis 2020;1-4:101665

21 Zambrano LI, Fuentes-Barahona IC, Bejarano-Torres DA, et al. A pregnant woman with COVID-19 in Central America. Travel Med Infect Dis 2020;36:101639

22 Gidlöf S, Savchenko J, Brune T, Josefsson H. COVID-19 in pregnancy with comorbidities: More liberal testing strategy is needed. Acta Obstet Gynecol Scand 2020;99(07):948-949

23 Kalafat E, Yaprak E, Cinar G, et al. Lung ultrasound and computed tomographic findings in pregnant woman with COVID-19. Ultrasound Obstet Gynecol 2020;55(06):835-837

24 Díaz CA, Maestro ML, Pumarega MTM, Antón BF, Alonso CP. First case of neonatal infection due to COVID-19 in Spain. An Pediatr (Engl Ed) 2020. Doi: 10.1016/j.anpede.2020.03.002

25 Alzamora MC, Paredes T, Caceres D, Webb CM, Valdez LM, La Rosa M. Severe COVID-19 during pregnancy and possible vertical transmission. Am J Perinatol 2020;37(08):861-865

26 Cao D, Yin H, Chen J, et al. Clinical analysis of ten pregnant women with COVID-19 in Wuhan, China: a retrospective study. Int J Infect Dis 2020;95:294-300

27 Chen H, Guo J, Wang C, et al. Clinical characteristics and intrauterine vertical transmission potential of COVID-19 infection in nine pregnant women: a retrospective review of medical records. Lancet 2020;395(10226):809-815

28 Chen S, Liao E, Cao D, Gao Y, Sun G, Shao Y. Clinical analysis of pregnant women with 2019 novel coronavirus pneumonia. J Med Virol 2020:1-6

29 Fan C, Lei D, Fang C, et al. Perinatal transmission of COVID-19 associated SARS-CoV-2: should we worry? Clin Infect Dis 2020: ciaa226

30 Khan S, Peng L, Siddique R, et al. Impact of COVID-19 infection on pregnancy outcomes and the risk of maternal-to-neonatal intrapartum transmission of COVID-19 during natural birth. Infect Control Hosp Epidemiol 2020;41(06):748-750

31 Liu Y, Chen H, Tang K, Guo Y. Clinical manifestations and outcome of SARS-CoV-2 infection during pregnancy. J Infect 2020:S01634453(20)30109-2

32 Qiancheng X, Jian S, Lingling P, et al;sixth batch of Anhui medical team aiding Wuhan for COVID-19. Coronavirus disease 2019 in pregnancy. Int J Infect Dis 2020;95:376-383

33 Xu L, Yang Q, Shi H, et al. Clinical presentations and outcomes of SARS-CoV-2 infected pneumonia in pregnant women and health 
status of their neonates. Sci Bull (Beijeng) 2020. Doi: 10.1016/j. scib.2020.04.040

$34 \mathrm{Yu} \mathrm{N}$, Li W, Kang Q, et al. Clinical features and obstetric and neonatal outcomes of pregnant patients with COVID-19 in Wuhan, China: a retrospective, single-centre, descriptive study. Lancet Infect Dis 2020;20(05):559-564

35 Zhu H, Wang L, Fang C, et al. Clinical analysis of 10 neonates born to mothers with 2019-nCoV pneumonia. Transl Pediatr 2020;9 (01):51-60

36 Breslin N, Baptiste C, Gyamfi-Bannerman C, et al. Coronavirus disease 2019 infection among asymptomatic and symptomatic pregnant women: two weeks of confirmed presentations to an affiliated pair of New York City hospitals. Am J Obstet Gynecol MFM 2020:1-7

37 Juusela A, Nazir M, Gimovsky M. Two cases of coronavirus 2019related cardiomyopathy in pregnancy. Am J Obstet Gynecol MFM 2020. Doi: 10.1016/j.ajogmf.2020.100113

38 Mulvey JJ, Magro CM, Ma LX, Nuovo GJ, Baergen RN. Analysis of complement deposition and viral RNA in placentas of COVID-19 patients. Ann Diagn Pathol 2020;46:151530

39 Ferrazzi E, Frigerio L, Savasi V, et al. Vaginal delivery in SARS-CoV2-infected pregnant women in Northern Italy: a retrospective analysis. BJOG 2020;127(09):1116-1121

40 Hantoushzadeh S, Shamshirsaz AA, Aleyasin A, et al. Maternal death due to COVID-19. Am J Obstet Gynecol 2020;223(01):109.e1-109.e16

41 Vlachodimitropoulou Koumoutsea E, Vivanti AJ, Shehata N, et al. COVID-19 and acute coagulopathy in pregnancy. J Thromb Haemost 2020;18(07):1648-165

42 Mullins E, Evans D, Viner RM, O'Brien P, Morris E. Coronavirus in pregnancy and delivery: rapid review. Ultrasound Obstet Gynecol 2020;55(05):586-592

43 Chawanpaiboon S, Vogel JP, Moller AB, et al. Global, regional, and national estimates of levels of preterm birth in 2014: a systematic review and modelling analysis. Lancet Glob Health 2019;7(01): e37-e46

44 Zeng H, Xu C, Fan J, et al. Antibodies in infants born to mothers with COVID-19 pneumonia. JAMA 2020;323(18):1848-1849

45 Kimberlin DW, Stagno S. Can SARS-CoV-2 infection be acquired in utero?: more definitive evidence is needed JAMA 2020;323(18): 1788-1789
46 Qiu L, Liu X, Xiao M, et al. SARS-CoV-2 is not detectable in the vaginal fluid of women with severe COVID-19 infection. Clin Infect Dis 2020. Doi: 10.1093/cid/ciaa375

47 Yu N, Li W, Kang Q, Zeng W, Feng L, Wu J. No SARS-CoV-2 detected in amniotic fluid in mid-pregnancy. Lancet Infect Dis 2020;1-2: S1473-3099(20)30320-0

48 Elshafeey F, Magdi R, Hindi N, et al. A systematic scoping review of COVID-19 during pregnancy and childbirth. Int J Gynecol Obstet 2020. Doi: $10.1002 /$ ijgo. 13182

49 Martínez-Perez O, Vouga M, Cruz Melguizo S, et al. Association between mode of delivery among pregnant women with COVID19 and maternal and neonatal outcomes in Spain. JAMA 2020;324 (03):296-299

50 Betrán AP, Ye J, Moller AB, Zhang J, Gülmezoglu AM, Torloni MR. The increasing trend in caesarean section rates: global, regional and national estimates: 1990-2014. PLoS One 2016;11(02): e0148343

51 American College of Obstetricians and Gynecologists. COVID-19 FAQs for Obstetrician-Gynecologists, Obstetrics. Updated March 26, 2020. Accessed April 30, 2020 at: https://www.acog. org/clinical-information/physician-faqs/covid-19-faqs-for-obgyns-obstetrics

52 Poon LC, Yang H, Lee JCS, et al. ISUOG interim guidance on 2019 novel coronavirus infection during pregnancy and puerperium: information for healthcare professionals. Ultrasound Obstet Gynecol 2020;55(05):700-708

53 Royal College of Obstetricians \& Gynaecologists. Coronavirus (COVID-19) Infection in Pregnancy. Accessed April 30, 2020 at: https://www.rcog.org.uk/globalassets/documents/guidelines/ 2020-04-17-coronavirus-covid-19-infection-in-pregnancy. pdf2020

54 Saccone G, Florio A, Aiello F, et al. Psychological impact of coronavirus disease 2019 in pregnant women. Am J Obstet Gynecol 2020;223(02):293-295

$55 \mathrm{Wu} \mathrm{Y,} \mathrm{Zhang} \mathrm{C,} \mathrm{Liu} \mathrm{H,} \mathrm{et} \mathrm{al.} \mathrm{Perinatal} \mathrm{depressive} \mathrm{and} \mathrm{anxiety}$ symptoms of pregnant women along with COVID-19 outbreak in China. Am J Obstet Gynecol 2020. Doi: 10.1016/j.ajog.2020.05.009

56 Thapa SB, Mainali A, Schwank SE, Acharya G. Maternal mental health in the time of the COVID-19 pandemic. Acta Obstet Gynecol Scand 2020;99(07):817-818 